\title{
Diagnosis of ventricular tachycardia: a clinical algorithm
}

\author{
MARK DANCY， DAVID WARD
}

Differentiating ventricular tachycardia from other broad complex tachycardias is important both for the management of the acute arrhythmia and for long term treatment to prevent its recurrence. In our experience ventricular tachycardia is often mistaken for supraventricular tachycardia, and clinical bias in favour of supraventricular tachycardia is strong. ${ }^{\text {Ia }}$

Several arrhythmias may give rise to broad complex tachycardias - ventricular tachycardia, supraventricular tachycardia with rate related aberrant conduction (including nodal tachycardia, atrial tachycardia, and reciprocating tachycardia associated with an accessory pathway), and any supraventricular tachycardia in a patient with pre-existing bundle branch block or pre-excitation. Clinically the most important of these is ventricular tachycardia as it has a worse prognosis, ${ }^{1}$ and its acute management is different from that of supraventricular tachycardias-for example, verapamil may cause life threatening hypotension without terminating ventricular tachycardia. ${ }^{2}$ The algorithm is therefore designed to distinguish ventricular tachycardia from other broad complex tachycardias. We recognise that in some cases arrhythmias cannot be diagnosed using the 12 lead electrocardiogram, and in these circumstances we suggest further, more specialised investigations. Furthermore, where the algorithm indicates ventricular tachycardia this implies only that the arrhythmia is highly likely to be ventricular tachycardia.

A bewildering number of features have been described as being valuable for differentiating broad complex tachycardias, and the algorithm is designed to bring together the most important of these features in a coordinated whole. We estimate that $95 \%$ of all cases of ventricular tachycardia can be correctly diagnosed by following this scheme.

The factors that are useful in distinguishing ventricular tachycardia from other forms of broad complex tachycardia are summarised in the figure. Apart from the specific features, described in more detail below, the patient may have an underlying cardiac disease of which the arrhythmia is an expression. Patients with ischaemic heart disease, especially those with left ventricular aneurysms, may be more likely to develop ventricular arrhythmias. Patients with thyrotoxicosis tend to suffer from atrial arrhythmias. Profound haemodynamic collapse is unusual in supraventricular tachycardia, ${ }^{3}$ although in all other respects the symptoms of supraventricular and ventricular tachycardia are similar.

Clinical features may show atrioventricular dissociation (the venous pulse rate may be less than the ventricular rate, or there may be variable intensity of the first heart sound). Although ventricular tachycardia may sometimes be diagnosed on purely clinical grounds, it is important to obtain an electrocardiographic tracing for confirmation. Unfortunately, in the excitement of treating

\footnotetext{
Cardiac Departments, St George's Hospital, London SW17, and Brompton Hospital, London SW3

MARK DANCY, MRCP, senior registrar

DAVID WARD, MRCP, senior registrar

Correspondence to: Dr Dancy.
}

patients with tachycardia sometimes no record is made of the tachycardia or only a short rhythm strip is recorded. This results in a serious loss of valuable information. Unless the patient is profoundly unwell a 12 lead electrocardiogram should be recorded in all cases of tachycardia and if possible it should be recorded continuously or brief strips taken every 30 seconds while any pharmacological intervention is undertaken. A rhythm strip can, however, give valuable information about the regularity of the rhythm and the atrioventricular relation (particularly if the lead with the largest $P$ wave deflection is recorded). The $R-R$ interval during ventricular tachycardia seldom varies more than $40 \mathrm{~ms}$, and if there is greater irregularity than this the rhythm is likely to be supraventricular, possibly atrial fibrillation with bundle branch block or pre-excitation. There are four main groups of electrocardiographic features that may be helpful (see figure).

\section{Clinical features of atrioventricular dissociation: \\ varying intensity of first heart sound \\ venous pulse rate less than arterial}

Electrocardiographic features of atrioventricular dissociation:

atrioventricular dissociation

blocked ventriculoatrial conduction

capture or fusion beats

QRS duration $>140 \mathrm{~ms}$

QRS axis more negative than $-30^{\circ}$

QRS morphology:

multiple morphologies

detailed analysis of QRS morphology?

Clinical and electrocardiographic features useful in distinguishing ventricular tachycardia from other forms of broad complex tachycardia.

\section{Atrioventricular dissociation}

Atrioventricular dissociation on the electrocardiogram may present in a number of guises, and it is a highly reliable indicator of ventricular tachycardia, although there have been a few recorded cases of nodal tachycardia with atrioventricular dissociation. ${ }^{4}$ Careful scrutiny of the electrocardiogram may reveal regular deflections at a rate unrelated to the $R-R$ interval. There may be capture or fusion beats. It is not uncommon for there to be retrograde (ventriculoatrial) conduction during ventricular tachycardia. If there is a one to one relation between $P$ waves and $Q R S$ complexes it is impossible to tell whether the $P$ wave is related to the preceding or succeeding QRS complex unless the initiating or terminating cycle of tachycardia has been recorded. Ventriculoatrial conduction is, however, sometimes blocked ( $2: 1,3: 1$, etc), in which case atrial activity cannot be initiating the tachycardia. Occasionally carotid sinus massage will increase the degree of ventriculoatrial 


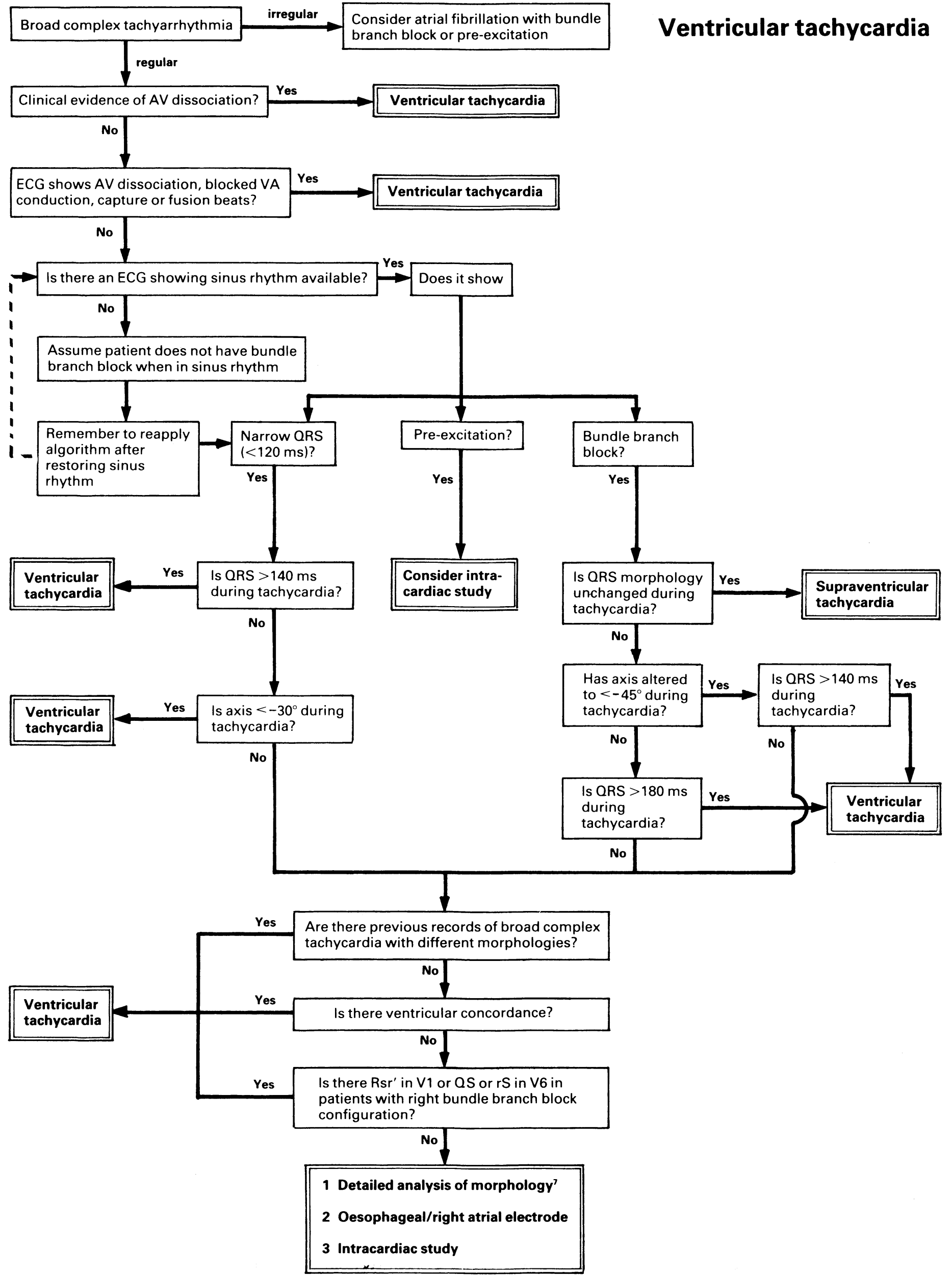


conduction block, ${ }^{5}$ making the diagnosis of ventricular tachycardia clear. Where there is doubt about the presence of atrioventricular dissociation, and the other electrocardiographic features do not allow a definitive diagnosis, the question can be resolved by simultaneous atrial and surface electrocardiographic recordings. This can either be carried out during tachycardia, or, if attacks are not sustained, by Holter monitoring with a two channel recorder, one channel of which is attached to a right atrial electrode. ${ }^{6}$ About $45 \%$ of patients with ventricular tachycardia may show electrocardiographic evidence of dissociated atrial activity.

\section{QRS duration and axis}

If there is no clinical or electrocardiographic evidence of atrioventricular dissociation the next line of approach is to examine the duration of the QRS complex. One of the problems associated with this and the consideration of QRS axis (see below) is that the sensitivity and specificity of the criteria depend on the presence or absence of bundle branch block during sinus rhythm. If there is preexisting bundle branch block different criteria need to be applied. This may present a problem when a patient is seen for the first time but once sinus rhythm is restored the tracing of tachycardia should be reviewed in the light of the further information provided by the 12 lead electrocardiogram of sinus rhythm. If there is no tracing of sinus rhythm available we assume for the purposes of this algorithm that the patient does not have pre-existing bundle branch block. This is most likely to be the case.

For those with a normal QRS duration during sinus rhythm an increase in duration to over $140 \mathrm{~ms}$ during tachycardia indicates ventricular tachycardia. ${ }^{7}$ In this group of patients (with narrow $Q R S$ in sinus rhythm) left axis deviation $\left(<-30^{\circ}\right)$ also suggests ventricular tachycardia.

In patients with pre-existing bundle branch block different criteria may be applied. Firstly, patients with bundle branch block usually retain the same morphology as they show in sinus rhythm if they develop supraventricular tachycardia. ${ }^{8}$ If the morphology changes during tachycardia the rhythm is likely to be ventricular tachycardia but various empirical combinations of axis change and QRS width may be used to improve specificity. ${ }^{\circ}$

An unusual cause of wide QRS in sinus rhythm is pre-excitation, and tachycardias in this group of patients are difficult to diagnose using the surface electrocardiogram. For this reason alone it may be necessary to proceed to intracardiac study, but there are additional reasons, such as measuring the refractory period and mapping the position of the accessory pathway, that increase the value of invasive studies in these patients. Although it is possible to hypothesise about the mechanism of tachycardia in patients with pre-excitation, one should have a low threshold for proceeding to intracardiac study.

\section{QRS morphology}

If the analysis of QRS duration and axis are unhelpful the QRS morphology may be distinctive. Firstly, in patients with supraventricular tachycardia with aberrant conduction the QRS morphology is usually the same with each episode (although aberrancy may be supplanted by normal conduction). On rare occasions patients with supraventricular tachycardia may show right bundle branch block on one occasion and left bundle branch block on another. ${ }^{10}$ The presence of more than two morphologies of tachycardia, or even only two morphologies if they are bizarre, indicates ventricular tachycardia. This requires a thorough search for all 12 lead electrocardiograms of tachycardia in the notes and relies on good documentation.

Ventricular concordance (uniformly positive or uniformly negative deflections across all the chest leads) is virtually diagnostic of ventricular tachycardia, although positive concordance may be seen in patients with type A pre-excitation. ${ }^{11}$ Detailed analysis of the morphology of individual leads according to the scheme of Wellens et al may add useful information, ${ }^{7}$ although on the whole the criteria are less specific. In our experience three morphologies that are specific appear sufficiently often to be worth memorising$\mathrm{Rsr}^{\prime}$ in V1 and QS or $\mathrm{rS}$ in V6 in patients with right bundle branch block morphology tachycardia. All three indicate ventricular tachycardia.

If the above scheme is followed, the vast majority of cases of broad complex tachycardia can be diagnosed without resort to intracardiac studies. If the diagnosis is still unclear it will be necessary to proceed either to more detailed documentation of atrial activity as mentioned above, or intracardiac studies with or without provocation.

\section{References}

1a Dancy M, Camm AJ, Ward D. Misdiagnosis of chronic recurrent ventricular tachycardia. Lancet 1985;ii:320-3.

1 Swerdlow CD, Winkle RA, Mason JW. Determinants of survival in patients with ventricular tachyarrhythmias. N Engl F Med 1983;308: 1436-42.

2 Sung RJ, Shapiro WA, Shen EN, Morady F, Davis J. Effects of verapamil on ventricular tachycardias possibly caused by reentry, automaticity, and triggered activity. $\mathcal{f}$ Clin Invest 1983;72:350-60.

3 Kapoor WN, Karpf M, Weisand S, Peterson JR, Levey GS. A prospective evaluation and follow up of patients with syncope. $N$ Engl I Med 1983;309:197-204.

4 Langendorf R. Differential diagnosis of ventricular tachycardia. Experimental Medicine and

Surgery 1959;8:228-39.
5 Hess DS, Hanlon T, Scheinman M, Budge R, Desai J. Termination of ventricular tachycardia by carotid sinus massage. Circulation 1982;65:627-33.

6 Lipkin DP, Perrins EJ, Schapiro LM, Fox KM. Diagnosis of broad complex tachycardias with ambulatory monitoring of atrial electrography. $\mathrm{BrMed} f$ 1984;228:1713-4.

Wellens HJ, Bar FWHM, Lie KI. The value of the electrocardiogram in the differential diagnosis of a tachycardia with a widened QRS complex. Am f Med 1978;64:27-33.

8 Dongas J, Joginder S, Denker S, Lehman M, Mahmud R, Akhtar M. Value of preexisting bundle branch block in the electrocardiographic differentiation of supraventricular from ventricular origin of wide QRS tachycardia. Am f Cardiol 1985;55:717-21.

9 Farre J, Hernandez R, Grande A, et al. New electrocardiographic observations in the differential diagnosis of tachycardias with a wide QRS complex. Role of electrophysiologic studies in the reevaluation of the diagnostic potential of the surface ECG [English Abstract]. Rev Esp Cardiol evaluation of the di

10 Wellens HJ, Ross DL, Farre J, Bruggada P. Functional bundle branch block during supraventricular tachycardia in man: observations on mechanisms and their incidence. In: Cardiac electrophysiology and arrhythmias. New York: Grune and Stratton, 1985.

11 Jackman WM, Friday KJ, Nacarelli GV. VT or not VT? An approach to the diagnosis and management of wide complex tachycardia. Clinical Progress in Pacing and Electrophysiology 1983;1:225-68.

(Accepled 26 fune 1985)

Is it safe to use slurry (sewage sludge) as a fertiliser on playing pitches? A patient is concerned that it is now proposed that the school rugby pitches should be covered with this material during the summer in readiness for next winter.

Sewage sludge contains a variety of bacterial, viral, protozoan, and metazoan pathogens that are viable for prolonged periods. Their concentration in sewage is about $1 / 1000$ th to $1 / 10000$ th that of faeces. Sewage sludge may be applied to land either directly from pipes or by spraying from a gun. Sludge may also be disposed of by compressing it into solid cakes and then depositing them on land. There are two obvious health risks from spraying. ${ }^{12}$ Firstly, pathogens may be carried by the aerosol to people in close proximity, and, secondly, the persistence of the organisms on the ground might lead to direct disease transmission. Sewage sludge should not be applied to any area that will be used for contact sport. ${ }^{3}-D$ BAXTER, lecturer in community medicine, Manchester.

1 Cronholm LS. Potential health hazards from microbial aerosols in densely populated urban regions. Applied and Environmental Microbiology 1980;39:6-12.

2 Hickey J, Reist P. Health significance of airborne microorganisms from wastewater treatment processes. Water Pollution Control Federation 1975;47:2741-57.

3 North West Water. Code of practice for the disposal of sewage sludge to agricultural land. Warrington: Department of Operations, 1983.

Elderly men with frequency find long distance bus journeys embarrassing. What advice should be given?

The best way to deal with this problem is to give the man a disposable plastic urine bottle. Downs Surgical plc (Church Path, Mitcham, Surrey CR4 3UE, tel: 016486291 ) make the Reddy-Bottle, which is ideal for this purpose. The user may carry it in his pocket until he needs to fill it. He can then pass urine. If he also takes a travel rug he can use it under the rug discreetly and dispose of it when convenient. It is so designed that it will not spill after use.- $\mathbf{R} E$ IRVINE, consultant physician, Hastings. 